\title{
Condiciones sociodemograficas y estado nutricional de niños menores de un año en areas perifericas de Guadalajara, México*
}

\author{
Sociodemographic conditions and nutritional status of children under one year \\ old in areas adjacent to Guadalajara, México
}

\author{
Guillermo Julián González P.**, María Guadalupe Vega L."*
}

\begin{abstract}
GONZALEZ P., G.J. \& VEGA L., M.G. Condiciones sociodemograficas y estado nutricional de niños menores de un año en areas perifericas de Guadalajara, México. Rev. Saúde Pública, 28: 268-76, 1994. Se objetivó determinar la proporción de niños menores de un año con peso inferior al mínimo adecuado para su edad, identificar factores de índole sociodemográfica que pudieran estar asociados a tal problemática, y estudiar la importancia sociosanitaria de los mismos, en áreas periféricas de la zona metropolitana de Guadalajara (ZMG), México. Se estudió una muestra probabilística, y representativa de niños menores de un año, nacidos entre el 1.5.1990 y 30.4.1991, e hijos de madres derechohabientes del Instituto Mexicano del Seguro Social, en Tlaquepaque y Tonalá, municipios conurbados con Guadalajara, segunda ciudad en importancia de México. La evaluación ponderal de los infantes se realizó a partir de las tablas que con tal fin ha elaborado el Centro Latinoamericano de Perinatología. Mediante el empleo de la regresión logística se estimaron Odds Ratios (OD), con intervalos de confianza (IC) del 90\%. El modelo hallado fue ajustado mediante el estadístico $\mathrm{H}^{*}$. Se calculó el riesgo atribuible poblacional (RAP), y la probabilidad de que un niño no alcanzara el peso mínimo adecuado (PMA) para su edad en presencia o ausencia de los factores de riesgo identificados. Los resultados muestran que casi la quinta parte de los lactantes estudiados no alcanzan el PMA. Se evidenció la asociación existente entre la edad de la madre menor de 20 años (OR=2,53, IC $1,03-6,22)$ y la pertenencia materna al grupo social "obrero" (OR=2,28, IC $1,01-5,21)$ con que el infante no alcance el PMA y la transcendencia social de tales factores (RAP $=26,4 \%$ y $36,7 \%$, respectivamente). En presencia de todos los factores analizados, la probabilidad de que un infante no alcance el PMA, es 0,85 , y en particular, en presencia solo de los factores de riesgo identificados, esta probabilidad es de 0,41. Esta información brinda pautas a los servicios de salud para diseñar acciones apropriadas dirigidas a grupos de población específicos con vistas a mejorar el estado nutricional de la población infantil.
\end{abstract}

Descriptores: Estado nutricional. Factores sociodemograficos. Salud infantil.

\section{Introducción}

Los problemas nutricionales han sido objeto de especial atención en las últimas décadas, tanto desde una perspectiva clínica como epidemiológica, dada la repercusión que los mismos tienen sobre el estado de salud de la población y la relevancia que alcanzan en los países subdesarrollados.

En México, en particular, diversos estudios $6,7,20$ se han encaminado a evaluar la situación nutricional de la población infantil, pues es evidente la trascendencia de la misma en los procesos de crecimiento y desarrollo del niño, en su morbimortalidad, y en la calidad de vida que éste posea cuando sea adulto.

* Presentada una versión en el "II Taller Latinoamericano sobre nutrición y salud en areas urbanas" México, DF., 1992.

* Instituto Regional de Investigación en Salud Publica de la Universidad de Guadalajara

Separatas/Reprints: G. J. Gonzáles P. Medrano 316, Sector Reforma. CP 44450. Guadalajara, Jalisco, México
Si bien distintos indicadores han sido utilizados para medir el estado nutricional del infante, la OMS ha seleccionado al peso como el más indicado para el seguimiento del lactante, sobre todo en los primeros meses de vida, pues es la dimensión antropométrica más sensible a los cambios nutricionales $^{10}$.

Así, pesos bajos para la edad - de acuerdo con el peso al nacer- durante el primer año de vida podrían estar evidenciando importantes carencias nutricionales.

La desnutrición es, sin duda, un problema latente en el contexto latinoamericano, que aqueja principalmente a la población menor de 5 años, y del cual México no está exento ${ }^{23}$. Como problema de salud ha sido examinado fehacientemente; sin embargo, los esfuerzos han estado dirigidos más a categorizar y clasificar el fenómeno que a indagar en la esencia del mismo. Aún cuando tácitamente se reconoce la determinación social de la nutrición humana - en la medida que responde, por un lado, a la capacidad social de producir alimentos, y por 
otro a la distribución socialmente diferenciada de los mismos en una comunidad determinada ${ }^{4}$ - los servicios de salud, al enfrentar los problemas nutricionales, tradicionalmente se han abstraído de ello, dedicandose más a la curación de la patología que a la exploración de sus causas, dejando a un lado las condicionantes socioeconómicas que la provocan para concentrarse en el enfermo que acude a las instituciones de salud.

Ciertamente, la solución de tales problemas rebasa los marcos del sector salud; sin embargo, el conocimento del contexto sociodemográfico en que estos se dan parece necesario con vistas a establecer estrategias de intervención que posibiliten la prevención de los mismos, o al menos su atención oportuna. El presente trabajo, llevado a cabo en áreas de la zona metropolitana de Guadalajara (ZMG), apunta hacia tal dirección; su propósito no es solo determinar la proporción de niños que no alcanzan un peso mínimo adecuado (PMA) para su edad, sino fundamentalmente establecer cuales podrían ser los factores de riesgo, de índole sociodemográfica, asociados a tal condición, y la trascendencia social de los mismos.

\section{Material y Metodo}

El presente estudio se realizó durante 1991 en los municipios de Tlaquepaque y Tonalá, los cuales se encuentran conurbados con la ciudad de Guadalajara. Los mismos forman parte de la ZMG, y aportan, entre ambos, aproximadamente el $18 \%$ de la población de esta urbe (capital del estado de Jalisco), que contaba, en 1990, con 2.870.417 habitantes. ${ }^{9}$

Para llevar a cabo la investigación, se seleccionó una muestra representativa de niños menores de un año, nacidos entre el $1.5 .1990 \mathrm{y}$ el 30.4.91, sobrevivientes al primer mes de vida, e hijos de madres derechohabientes del Instituto Mexicano del Seguro Social (IMSS) que residieran en los municipios antes mencionados. Aproximadamente 3.000 niños cumplían con estas condiciones y por lo tanto, constituyeron el universo de estudio.

El diseño muestral tuvo un carácter polietápico y probabilístico; en una primera fase se seleccionaron aleatoriamente, del total de Unidades de Atención Primaria a la Salud del IMSS existentes en los municipios estudiados -4-, aquellas que intervinieron en el estudio -2-, para luego elegir al azar, los consultorios que fueron elegidos para la investigación, 6 en total considerando ambas clínicas. En cada uno de estos consultorio se intentó estudiar a todos los niños que reunieran los criterios previamente establecidos, descritos en el párrafo anterior.

Si bien el número total de infantes a estudiar era 166, el tamaño definitivo de la muestra ascendió a 141, ya que por diversas causas - cambio de domicilio, no existencia del domicilio registrado, entre otras- no fue posible contactar con todos los niños. A las madres de los niños estudiados se les aplicó un cuestionario con diversos módulos preparados al efecto. Además, los niños fueron pesados y medidos al momento del estudio. Ambas tareas fueron desarrolladas por trabajadoras sociales especialmente capacitadas para ello.

En principio, se procedió al cálculo de diversos indicadores que, o reflejan el estado nutricio del infante, o estan íntimamente asociados al mismo: aumento de peso de la madre durante el embarazo, peso al nacer, duración de la lactancia materna y peso para la edad (al momento del estudio).

El peso para la edad fue evaluado a partir de las Tablas de Crecimiento Postnatal que con tal fin fueron elaboradas por Martell y Belitzky ${ }^{15}$, a partir de considerar el peso al nacer y la velocidad media de crecimiento del lactante. Con este método, resulta sencillo y rápido hacer un primer diagnóstico del crecimiento y el estado nutricio del infante, pues se basa en dos mediciones de su peso corporal. Así, pudo conformarse para el estudio la categoría de mayor interés: aquellos niños que no alcanzan el PMA para su edad, o sea, estan por debajo del percentil 10 en las curvas tradicionales que relacionan peso con edad.

Con vistas al análisis estadístico, el no alcanzar el PMA para la edad fue considerado como la variable dependiente, evaluandose como variables predictoras o explicativas un conjunto de factores que de una u otra forma reflejan las condiciones socioeconómicas y demográficas que caracterizan a la madre y al niño: condiciones de vivienda muy deficientes (CV); edad materna menor de 20 años (EM); escolaridad de la madre menor de 6 años (AESC); pertenencia de la madre a grupo social "obrero" (POSOC); ingreso percápita familiar inferior a medio salario mínimo (IP); peso al nacer menor de 3.000 gramos (PN); duración de la lactancia materna menor de dos meses (LM); no conceptualización por parte de la madre de la alimentación como aspecto esencial para que el niño esté sano (NECSAN); hijo no deseado (HD).

Para evaluar las condiciones de vivienda, se tomaron en cuenta elementos tales como: hacinamiento (o sea, más de tres personas en promedio por habitación destinada exclusivamente a dormir), tenencia de agua entubada o servicios sanitarios dentro de la vivienda, presencia de equipos electrodomésticos en la misma, entre otros. Por otra parte, al momento del estudio el salario 
mínimo en la ZMG ascendía aproximadamente a 330.000 pesos mexicanos mensuales (unos 110 USD al cambio oficial vigente a la fecha). Por último, los valores que tomo la variable NECSAN dependieron de las respuestas que las madres brindaron a las preguntas" "¿Que cree usted que su hijo necesita para estar sano?" y "De todas las cosas que usted hace por su hijo, ¿cuál considera la más importante?"; se partió del criterio de que la no inclusión de la alimentación en ninguna de las dos respuestas ubicaba a la madre entre aquellas que no la conceptualizaban como un elemento básico para que el infante estuviera sano.

Las variables predictoras estudiadas fueron seleccionadas de un conjunto más amplio de variables; se incluyeron en el análisis solo aquellas que mostraron una mayor asociación estadística con la variable dependiente. A partir del criterio de que la exposición de los lactantes a estos factores podía modificar sustancialmente su situación nutricional, los mismos fueron considerados en el estudio como posibles factores de riesgo para que el infante alcanzara o no el PMA para su edad.

Para facilitar el análisis de la información, tanto las variables predictoras como la dependiente fueron dicotomizadas; por una parte se asignaron los valores 0 y 1 en ausencia o presencia del factor de riesgo, respectivamente; por otra, los valores 0 y 1 se adjudicaron de acuerdo a si el niño alcanzaba o no el PMA.

$\mathrm{El}$ análisis estadístico de la información se realizó mediante el empleo de la regresión logística; tal modelo permite estimar, tanto de forma univariada como multivariada, los "Odds Ratios" (OR) correspondientes a cada factor analizado; a través del programa LOGIT ${ }^{11}$, se corrieron diversos modelos de regresión logística, eligiendose aquel que presentaba un mejor ajuste para calcular los OR. La validez estadística de los modelos fue evaluada mediante la razón de verosimilitud y la prueba de chi cuadrado; los modelos se validaron mediante la prueba de bondad de ajuste de Hosmer y Lemeshow ${ }^{12}$ y en particular, estimando el estadístico $\mathrm{H}^{*}$. Los coeficientes de regresión b se estimaron mediante el método de máxima verosimilitud, y su nivel de significancia estadística se evaluó con la prueba de Wald, con o estadístico t de Student. El cálculo del exponencial de b permitió obtener los OR. Para los OR se estimaron intervalos de confianza del $90 \%$. Esto permitió aceptar o rechazar la hipótesis nula subyacente en el estudio, es decir, que no existía asociación entre las variables predictoras y la variable dependiente.

El empleo del nivel de confianza antes mencionado - relativamente aceptado en el campo de las ciencias sociales $^{13}$ - está íntimamente vinculado con las características del problema que se estudia: dada su naturaleza social, parecía más indicado sacrificar un poco la confianza estadística, en aras de no obviar factores presumiblemente asociados en cierto grado con la variable dependiente, y cuya identificación pudiera resultar relevante para los servicios de salud.

A partir del modelo definitivamente elegido - el que presentaba un mejor ajuste -, y mediante la expresión:

$$
p=\frac{1}{1+\exp \left\{-\alpha-\beta_{1} x_{1}-\ldots \beta_{k} x_{k}\right\}}
$$

donde $B 1 \ldots$...B son los coeficientes de regresión calculados para cada variable, y $\mathrm{x}_{1} \ldots \mathrm{x}_{\mathrm{k}}$ los valores que asume cada variable ( 1 cuando el infante está expuesto al factor de riesgo, 0 cuando no lo está), se calcularon las probabilidades de que un niño no alcanzara el PMA estando expuesto o no a los factores de riesgo identificados.

Indudablemente, el uso de la regresión logística se ha extendido en los últimos años en el campo de la epidemiología; de ahí que el desarrollo teórico del modelo, y distintas aplicaciones del mismo, pueden ser encontradas en diversos trabajos recientes $4,8,21,24$.

Además, la trascendencia social de los OR hallados fue evaluada mediante el cálculo del Riesgo Atribuíble en la Población (RAP) -a través de la fórmula de Levin ${ }^{14}$, para lo cual se tuvo en cuenta la proporción de niños de la muestra expuesta al factor de riesgo estudiado.

\section{Resultados}

En la Tabla 1 se presenta información referida a algunas características socioeconómicas y demográficas de la población estudiada; como puede observarse, los niños de la muestra provienen, en una alta proporción, de familias obreras, con in-

Tabla 1 - Características socioeconómicas y demográficas seleccionadas de la población estudiada. Valores absolutosy relativos. Tlaquepaque-Tonalá, ZMG. México, 1991.

\begin{tabular}{lcc}
\hline Variable & Ne & $\begin{array}{c}\% \text { del total } \\
n=141\end{array}$ \\
\hline Codiciones de vivienda muy deficientes & 15 & 10,6 \\
Escolaridad matema menorde 6años & 37 & 26,2 \\
Edad de la madre menorde 20 años & 33 & 23,4 \\
Madrespertenecientesagnuposocial obrero & 69 & 48,9 \\
$\begin{array}{l}\text { Ingreso percápita familiar menor a medio } \\
\text { salario mínimo }\end{array}$ & 66 & 46,8 \\
Hijono deseado & 24 & 17,0 \\
No conceptualización de la alimentación & & \\
como elemento básico para que el niño & & \\
esté sano & 31 & 21,3 \\
\hline
\end{tabular}


gresos percápitas mensuales inferiores a medio salario mínimo; uno de cada diez, aproximadamente, tiene condiciones de vivienda que pueden considerarse como muy deficientes.

Por otro lado, prácticamente la cuarta parte de las madres analizadas tuvieron su niño con menos de 20 años, y una proporción ligeramente superior no alcanzó a terminar sus estudios primarios; una de cada seis, al momento de quedar embarazada, no deseaba tener su niño, y algo más de la quinta parte no conceptualizaba a la alimentación como elemento básico para que su hijo estuviera sano.

La Tabla 2 permite apreciar el comportamiento de distintos indicadores que describen la situación nutricional de la madre y el infante. En primer lugar, puede observarse ue una notable proporción de madres (aproximadamente cuatro de cada diez) aumentó menos de $10 \mathrm{~kg}$ durante el embarazo; algo más de la quinta parte de los niños nacieron con bajo peso o con peso insuficiente (con menos de 2.500 gramos, o entre 2.500 y 2.999 gramos, respectivamente); y casi la mitad de los recien nacidos recibieron lactancia materna por un lapso inferior a los dos meses.

Tabla 2 - Indicadores nutricionales seleccionados de la madre y el niño, en la población estudiada. Valores absolutos y relativos. Tlaquepaque-Tonalá, ZMG. México, 1991.

\begin{tabular}{lcc}
\hline Indicadores & $\mathrm{N}^{2}$ & $\begin{array}{c}\% \text { del total } \\
n=141\end{array}$ \\
\hline Aumento de peso de la madre durante el & & \\
embarazo < 10 Kg. & 57 & 40,4 \\
Bajo peso al nacer $(<2.500 \mathrm{gr})$ & 6 & 4,3 \\
Pesoal nacer insuficiente (2.500-2.999 gr) & 25 & 17,7 \\
Lactancia materna inferior a dos meses & 68 & 48,2 \\
Niños que no alcanzan el peso minimo a- & & \\
decuado(PMA) parala edad & 26 & 18,4 \\
\hline
\end{tabular}

En especial, al evaluar el estado nutricional de los niños al momento del estudio, se encontró que casi la quinta parte de los mismos no alcanzaba el PMA de acuerdo a su edad.

Los resultados del análisis de regresión logística se muestran en las Tablas 3 y 4 ; en el análisis univariado se pone de manifiesto que es más riesgoso, para que el niño no alcance el PMA, estar expuesto a las condiciones consideradas como posibles factores de riesgo, que no estarlo; en la mayoría de los casos puede hablarse de la existencia, entre cada factor por separado y la variable dependiente, de una asociación estadísticamente significativa.

$\mathrm{El}$ análisis multivariado, por su parte, ofrece resultados diferentes, tanto en el orden de importancia como en el grado de asociación de las variables predictoras con el hecho de que los infantes no alcancen el PMA para su edad. Desde esta pers-
Tabla 3 - Asociación existente entre no alcanzar el peso mínimo adecuado para la edad y variables seleccionadas: Resultados de la regresión logística, análisis univariado. Tiaquepaque-Tonalá, ZMG. México, 1991.

\begin{tabular}{lcccc}
\hline Variables & $\begin{array}{c}\text { Coeficiente } \\
\beta\end{array}$ & $\begin{array}{c}\text { Error } \\
\text { Estandar }\end{array}$ & OR & $\begin{array}{c}1 \mathrm{C} \\
(90 \%)\end{array}$ \\
\hline CV & .8872 & .1870 & $2,43^{*}$ & $1,79-3,30$ \\
EM & .9249 & .1999 & $2,52^{*}$ & $1,81-3,50$ \\
AESC & .6485 & .2057 & $1,91^{*}$ & $1,36-2,68$ \\
POSOC & .7210 & .2282 & $2,06^{*}$ & $1,41-2,99$ \\
IP & .3360 & .2208 & 1,40 & $0,97-2,01$ \\
PNAC & .3859 & .2083 & $1^{*}, 47^{*}$ & $1,04-2,07$ \\
LM & .4602 & .2219 & $1,58^{*}$ & $1,09-2,28$ \\
NECSAN & .6326 & .2039 & $1,88^{*}$ & $1,35-2,63$ \\
HD & .7550 & .1972 & $2,12^{*}$ & $1,53-2,94$ \\
\hline
\end{tabular}

OR-Odds Ratio

IC-Intervalo de Confianza

* - Significación Estadística $p<0,1$

Tabla 4 - Asociación existente entre no alcanzar el peso minimo adecuado para la edad y variables seleccionadas: Resultados de la regresión logística, análisis univariado. Tlaquepaque-Tonalá, ZMG. México, 1991.

\begin{tabular}{lccccc}
\hline Variables & $\begin{array}{c}\text { Coeficiente } \\
B\end{array}$ & $\begin{array}{c}\text { Error } \\
\text { Estandar }\end{array}$ & $\begin{array}{c}\text { Prob. } \\
(\mathbf{t})\end{array}$ & & $\begin{array}{c}\text { OR } \\
(90 \%)\end{array}$ \\
\hline CV & .3534 & .7844 & .50 & 1,42 & $0,39-5,17$ \\
EM & .9275 & .5473 & .09 & $2,53^{*}$ & $1,03-6,22$ \\
AESC & .3517 & .5716 & .50 & 1,42 & $0,56-3,64$ \\
POSOC & .8263 & .4875 & .09 & $2,28 *$ & $1,01-5,21$ \\
IP & .1095 & .5160 & .50 & 1,12 & $0,48-2,61$ \\
PNAC & .3195 & .5478 & .50 & 1,38 & $0,56-3,39$ \\
LM & .3659 & .5069 & .47 & 1,44 & $0,62-3,38$ \\
NECSAN & .5901 & .5177 & .25 & 1,80 & $0,77-4,23$ \\
HD & .8835 & .5846 & .13 & 2,42 & $0,93-6,33$ \\
Constante & .3 .0181 & .6242 & .00 & & \\
\hline RR Ring
\end{tabular}

RR-Riesgo Relativo

IC - Intervalo de Confianza

* Significación Estadística 0,1

Pruebax $^{2}$ ( $9 \mathrm{GL}$ ) de la razón de verosimilitud $=14,73 ; p<0,1$

pectiva, sale a relucir la relación intrínseca de cada factor con la variable dependiente, y el OR se interpreta como aquel que se obtendría si todos los demás fueran constantes.

Tanto en el análisis univariado como en el multivariado cabe señalar que todas las variables evaluadas como posibles factores de riesgo presentan un coeficiente positivo, y por lo tanto, exhiben un OR superior a 1 , lo que evidencia que, en sentido general, es más riesgoso para el estado nutricio del infante estar expuesto a cualquiera de las condiciones desfavorables evaluadas, que no estarlo. Esto habla a favor del proceso de selección de las variables incluídas en el modelo.

Entre las variables analizadas, fueron la edad de la madre $(<20$ años) y la posición social de la madre ( pertenencia a grupo social obrero) aquellas que mostraron una may or asociación - estadísticamente significativa - con la variable de- 
pendiente; así, ser hijo de madre con estas características eleva en forma notoria el riesgo del infante de no alcanzar el PMA para su edad, pues el OR más que duplica el de aquellos niños hijos de madres de 20 años o más, o provenientes de familias no obreras.

El hecho de que el límite inferior del intervalo de confianza sea menor y mayor de 1 , y los resultados de la prueba t, permiten en este caso, rechazar la hipótesis nula, según la cual, no existe asociación entre las variables explicativas y la variable dependiente. De los modelos de regresión logística construídos, este fue el que presentó un mejor ajuste; los resultados de la prueba de bondad de ajuste realizada $\left(\mathrm{H}^{*}=3,77\right.$-distribución chi cuadrado con 9 grados de libertad-, $P=0,5$ ), reflejan que el modelo se ajusta adecuadamente a los datos observados.

El OR estimado para la variable EM es similar en ambos análisis; llama la atención, sin embargo, que mientras en el análisis multivariado aumenta el OR de la variable "pertenencia de la madre a grupo social obrero", disminuye sustancialmente el de las variables CV, AESC e IP. Esto sugiere pensar que en el análisis univariado estas variables estaban jugando un papel confusor, y el efecto real de la variable POSOC se hallaba subsumido en ellas.

Resalta, además, el alto OR estimado para la variable "hijo no deseado", aún cuando, dado que el límite inferior del intervalo de confianza es menor a 1 , no pueda considerarse tal variable definitivamente como factor de riesgo.

Tabla 5 - Riesgo atribuible poblacional de las variables identificadas como factores de riesgo para que el niño no alcance el peso mínimo adecuado para la edad. Tlaquepaque-Tonalá, ZMG. México, 1991.

\begin{tabular}{lccc}
\hline $\begin{array}{l}\text { Factores de } \\
\text { Riesgo }\end{array}$ & $\begin{array}{c}\text { Proporcióndeniños } \\
\text { estudiados expuestos } \\
\text { al factor (\%) }\end{array}$ & $\begin{array}{c}\text { ODDS } \\
\text { Ratios }\end{array}$ & $\begin{array}{c}\text { Riesgo } \\
\text { Atribuible } \\
(\%)\end{array}$ \\
\hline $\begin{array}{l}\text { Edad de la ma- } \\
\text { dre<20 años }\end{array}$ & 23,4 & 2,53 & 26,4 \\
$\begin{array}{l}\text { Madre pertene- } \\
\text { ciente a grupo } \\
\text { socialobrero }\end{array}$ & 48,9 & 2,28 & 36,7 \\
\hline
\end{tabular}

En la Tabla 5 se brindan los Riesgos Atribuíbles hallados, para aquellas variables identificadas como factores de riesgo, o que presentan un alto OR; dado que el RAP depende no solo del OR, sino sobre todo de la proporción de infantes expuestos al factor de riesgo, es lógico que los valores más elevados los presente la pertenencia de la madre al grupo sociat "obrero", pues casi la mitad de los niños posee esta condición: la misma es responsable de algo más de la tercera parte de la
Tabla 6 - Probabilidad que tendria un niño de no alcanzar el peso mínimo adecuado para su edad, de acuerdo con la presencia o ausencia de los factores de riesgo identificados. Tlaquepaque-Tonalá, ZMG, México, 1991.

\begin{tabular}{lc}
\hline Factores de Riesgo & Probabilidad \\
\hline $\begin{array}{ll}\text { En presencia de todos los factores de riesgo } \\
\text { estudiados }\end{array}$ & 0,85 \\
$\begin{array}{l}\text { En presencia de los factores de riesgo iden- } \\
\text { tificados como tales (EM, POSOC) }\end{array}$ & 0,41 \\
$\begin{array}{l}\text { En ausencia de los factores de riesgo identi- } \\
\text { ficados }\end{array}$ & 0,05 \\
$\begin{array}{l}\text { Probabilidad real de no alcanzar el peso } \\
\text { mínimoadecuado de acuerdoalestudio }\end{array}$ & 0,18 \\
\hline
\end{tabular}

proporción de niños que no alcanzan el PMA, mientras que el resto de los factores evaluados apenas rebasan la cuarta parte (edad de la madre menor de 20 años) o la quinta (hijo no deseado).

En la Tabla 6 se exponen las probabilidades que tendría un niño de pesar menos del PMA para su edad, de acuerdo con su exposición o no a los factores estudiados. Si se tiene en cuenta que la probabilidad real de un infante de la muestra de no alcanzar el PMA es de 0,18, es evidente que la misma aumentaría considerablemente - hasta 0,85 - si tal niño estuviera expuesto a todas las circunstancias adversas que reflejan las variables incluídas en el modelo (condiciones de vivienda muy deficientes, madre con menos de 6 años de escolaridad, menor de 20 años, de posición social obrera, etc.).

Si el infante solo estuviera expuesto a las variables identificadas como más asociadas con el hecho de no alcanzar el PMA (ser hijo de madre menor de 20 años, de posición social obrera, o hijo no deseado) la probabilidad, aunque menor, seguiría siendo relativamente alta - 0,41 -; por el contrario, de no estar expuesto a ninguno de los factores estudiados, la probabilidad de que el lactante no logre el PMA sería mínima, 0,05.

\section{Discusión}

El IMSS brinda atención a una proporción importante de la población mexicana, y en particular, a más de la mitad de los habitantes de Jalisco. De ahí que un elevado número de nacimientos se produzca en sus instituciones, y que sea parte esencial de los servicios de salud del país.

Es una creencia generalizada que la población derechohabiente del IMSS posee condiciones de vida - y en particular de salud-mejores que las que tiene el resto de la población, sobre todo aquellos que no tienen acceso ni a los servicios que brinda el IMSS ni a la medicina privada. Si bien esto es relativamente cierto - el aseguramiento implica, 
por ejemplo, que al menos algún miembro de la familia posea un empleo más o menos estable-, la información obtenida en este estudio pone de manifiesto la heterogeneidad de la población derechohabiente; así, es posible encontrar en las áreas periféricas de la ZMG un número importante de familias que vivem en condiciones de vivienda muy deficientes o con bajos ingresos percápitas mensuales, un alto porcentaje de madres con menos de 20 años o que no alcanzan a terminar la educación primaria, entre otros aspectos. Esta disparidad sociodemográfica que presenta la población estudiada sin dudas ayuda a explicar el por qué de sus problemas nutricios.

Una visión de conjunto de los indicadores nutricionales hallados refleja la existencia de problemas que abarcan desde el embarazo - en donde la ganancia de peso de la madre en un notable número de casos no alcanza al menos $10 \mathrm{Kgs.}$, aumento deseado para evitar fallas ulteriores en el crecimiento del lactante ${ }^{21}$ - hasta el estado nutricio del menor de un año. Algunas investigaciones en México han evidenciado un aumento de peso significativamente menor durante el embarazo entre madres adolescentes con respecto a madres adul$t^{5}{ }^{5}$; el relativamente alto porcentaje de madres menores de 20 años en el estudio puede estar relacionado con los resultados encontrados.

Las dificultades en la ganancia de peso durante la gestación van a tener su reflejo más inmediato en la proporción relativamente alta encontrada de niños nacidos con bajo peso, o con peso insuficiente, más relevante aún si se tiene en cuenta que solo se estan estudiando infantes sobrevivientes al primer mes; es notoria tambien la práctica reducida de la lactancia materna en el grupo estudiado; es de sobra conocida la repercusión de este hecho en la situación nutricia del infante $^{18}$, y por ende, en su crecimiento y desarrollo adecuado.

Todo lo anterior conduce a pensar que los valores hallados en cuanto al estado nutricio del infante al momento del estudio no son fruto del azar, sino responden a la compleja realidad social en que estan insertos estos niños.

Las cifras encontradas de infantes por debajo del PMA para su edad son comparables con la proporción de desnutridos en edad preescolar, y en particular, en menores de un año, reportadas en otros estudios desarrollados en Latinoamérica ${ }^{1,2,16}$. La magnitud del problema, sin embargo, no deja de ser preocupante, sobre todo si se tiene en cuenta que la literatura en torno al tema refiere que las alteraciones del crecimiento son más evidentes según transcurre la edad del niño, en especial a partir del primer año de vida²; que alrededor de 500 lactantes de las áreas estudiadas, hijos de ma- dres derechohabientes del IMSS, presenten tal condición nutricional durante su primer año de vida es, sin dudas, un problema de salud pública que necesita respuestas rápidas y eficaces.

Si bien el estado nutricio del recién nacido va a responder, en lo inmediato, a aspectos tales como el aumento de peso de la madre durante el embarazo, el peso al nacer, o el amamantamiento materno, tanto estos indicadores como la propia situación nutricional estan condicionados - como evidencian diversos trabajos $3,5,17$ - por el contexto socioeconómico en que se desenvuelve el niño, y los atributos demográficos y sociales que lo caracterizan a él y a su familia.

En tal sentido, parece obvia la importancia que pudiera tener para los servicios de salud el poder establecer con antelación y oportunidad cuáles son los factores que influyen en que el niño no alcance el PMA correspondiente a su edad, pues a partir de tal información se pudieran trazar estrategias pertinentes para abordar tal problemática.

La asociación hallada desde el punto de vista estadístico, tras el análisis multivariado, entre diversas variables y el hecho de que el niño no logre el PMA pone de manifiesto, por una parte, la trascendencia de la maternidad adolescente para la condición nutricia del infante; por otra, la desventaja que enfrentan los hijos de obreros con respecto a otros sectores sociales en lo que a nutrición se refiere.

En el primero de los casos, la inexperiencia en el cuidado de los hijos, la falta de una identificación plena con el rol materno, y la inmadurez biológica, son entre otros, aspectos que repercuten, en distinto grado, en el crecimiento adecuado del infante, e inciden ya desde la propia gestación. La pertenencia al grupo social obrero, por otro lado, puntualiza una ubicación social que resume una serie de condiciones adversas de diversa índole, las cuales se reflejan tanto en la capacidad de adquisición de productos alimenticios como en hábitos alimentarios no satisfactorios, y se traducen en los problemas nutricionales previamente enunciados.

Un comentario particular merece la asociación entre la variable "hijo no deseado" y el hecho de que el lactante no tenga el PMA al momento del estudio. A pesar de que la asociación hallada en el estudio no es estadísticamente significativa, su magnitud deja entrever la necesidad de investigar con más profundidad esta relación, que indudablemente podría influir en los cuidados que la madre dedica a su hijo, y por tanto, en su situación nutricional.

La verdadera relevancia social de los factores de riesgo identificados en el estudio viene dada, esencialmente, por la cantidad de niños que se hal- 
lan expuestos a los mismos. Es evidente la importante reducción que en la prevalencia del fenómeno estudiado se pudiera alcanzar si los lactantes no estuvieran expuestos a los factores ya señalados, sobre todo en el caso de la pertenencia de la madre al grupo social obrero.

Lógicamente, no puede pensarse en la eliminación de tales factores de riesgo, pues, en efecto, sería absurdo esperar que las madres con esas características no parieran; no obstante, es factible disminuír el exceso de riesgo que acompaña a estos niños (o sea, reducir la influencia del factor), o incluso, en el caso de la edad materna, trasladar los nacimientos hacia edades más adecuadas, lo que indirectamente beneficiaría tanto la salud de la madre como la del infante, en especial su estado nutricio.

Lo anterior rebasa, definitivamente, el campo de acción de los servicios de salud e implica la incorporación de distintos sectores sociales a la búsqueda de soluciones a los problemas nutricionales que aquejan a la población estudiada.

No significa esto, sin embargo, que los prestadores de servicios sanitarios, y en este caso el IMSS, no puedan desempeñar un papel trascendente en la prevención y atención a este tipo de problemas. La estimación de la probabilidad de que el infante no alcance el PMA para su edad en tanto esté expuesto a los factores de riesgo identificados permite detectar con oportunidad - y con cierto grado de certeza-aquellos lactantes que podrían tener más probablemente situaciones nutricias comprometidas, $y$ por tanto, tomar decisiones eficaces para evitarlas. Así, los hijos de madres menores de 20 años, o que pertenecen al grupo social obrero, deberían ser objeto de un particular y oportuno cuidado desde el nacimiento, con vistas a prevenir posibles problemas nutricionales.

De tal forma, el modelo hallado tiene un importante valor predictivo en el plano individual, $y$, a su vez, las acciones derivadas del mismo podrían repercutir favorablemente en la disminución del porcentaje de niños que no alcanzan el PMA para su edad.

Desde una perspectiva macrosocial, la estimación de la probabilidad en presencia o ausencia de los factores de riesgo analizados posibilita visualizar en qué medida es reducible la proporción de niños que no alcanzan el PMA para su edad, si se superaran las condiciones sociodemográficas desventajosas a las que estan expuestos dichos infantes, y en particular, aquellas más relevantes. Si se observa que la proporción de niños sin el PMA para su edad calculada en el estudio, 0,18 , triplica la probabilidad de que un niño no alcance el PMA cuando no está expuesto a circunstancias desfavo- rables $(0,05)$, se evidencia cuánto es posible hacer aún - por parte de los servicios de salud, y por el sistema social en general- para lograr disminuir proporcionalmente el número de niños con este problema.

\section{Conclusiones}

Aún cuando el estudio presenta algunas limitaciones, como la delimitación expresa del mismo a población derechohabiente del IMSS, el tamano definitivo de la muestra, los lógicos sesgos en las respuestas a los cuestionarios utilizados, o el no emplear un nivel de significación estadística más riguroso - aspectos estos que pudieran repercutir en el alcance y validez de los resultados-, la información obtenida permite llegar a consideraciones importantes sobre la problemática analizada.

Existen problemas reales referidos a aspectos nutricionales en la población estudiada, que se reflejan en el elevado porcentaje de madres que aumentan menos de $10 \mathrm{~kg}$ durante el embarazo, la alta proporción de recién nacidos con peso no favorable, o que no son amamantados por un lapso prolongado, y en especial, el notable número de niños que no alcanza el PMA para la edad.

Se identificarón como factores de riesgo para que el infante no alcance el PMA, que la madre fuera menor de 20 años, y que perteneceria al grupo social obrero.

Cualquier política institucional para reducir la magnitud del problema en las áreas estidiadas debe tomar en cuenta estos factores - la posición social y la edad materna-, dada la importante proporción de niños que provienen de familias obreras o de madres adolescentes.

Para el pronóstico individual de problemas nutricios en el lactente, deben ser consideradas las diversas condiciones sociodemográficas que rodean a la madre y al niño, en particular aquellas cuya asociación con la no obtención de un PMA para la edad es manifiesta; esto posibilitaría acciones oportunas y eficaces favorables a la salud infantil.

Las deficiencias nutricionales de la población menor de un año en las áreas urbanas estudiadas son, sin dudas, un problema de salud pública, socialmente condicionado, cuya solución rebasa los marcos institucionales del sector salud; así, se requiere del esfuerzo mancomunado de diferentes actores sociales si se pretende que las nuevas generaciones tengan un desarrollo físico y neuromotor adecuado, y por ende, una mejor calidad de vida.

GONZALEZ P., G.J. \& VEGA L., M.G. Condiçōes sociodemográficas e estado nutricional de crianças me- 
nores de um ano em áreas periféricas da cidade de Guadalajara, México. Rev. Saúde Pública, 28: 268-76, 1994. Foi estudada a proporção de crianças com menos de um ano com peso inferior ao mínimo adequado para sua idade, identificados os problemas de natureza sociodemográfica que pudessem estar associados ao problema e a importância sócio-sanitária dos mesmos, em áreas da periferia da zona metropolitana de Guadalajara (ZMG), México. A pesquisa foi feita em uma amostra representativa de crianças nascidas entre 1.5.1990 (primeiro de maio de 1990) e 30.4 .91 (trinta de abril de 1991) em filhos de mães associadas ao Instituto Mexicano de Seguro Social nos municípios de Tlaquepaque e Tonalá, cidades satélites de Guadalajara, a segunda do país. A avaliação ponderada das crianças se fez a partir das tabelas feitas com esse propósito pelo Centro Latino-Americano de Perinatologia. Com o emprego da regressão logística, se estipularam "Odds Ratios" (OR), com intervalos de confiança (IC) de $90 \%$. O modelo encontrado foi ajustado mediante a estatística $\mathrm{H}^{*}$. Da mesma forma calculou-se o risco populacional atribuível (RPA), e a probabilidade de que a criança não atingisse o peso mínimo adequado (PMA) para sua idade na presença ou ausência dos fatores de risco identificados. Os resultados mostraram que quase uma quinta parte dos lactantes estudados não alcançam o PMA. Evidenciou-se associação existente entre a idade da mãe de menos de 20 anos $(O R=2,53$; IC $1,03-6,22$ ) e o fato de que pertença ao grupo social "operário" (OR= 2,28; IC 1,01-5,21) com o de que a criança não atingia o PMA e a transcendência social de tais fatores (RPA $=26,4 \%$ e $36,7 \%$ respectivamente). $\mathrm{Na}$ presença de todos os fatores analisados, a probabilidade de que uma criança não atinja o PMA é de 0,85 e, em particular, na presença so dos fatores de risco identificados, esta probabilidade é de 0,41. Esta informação fornece dados aos serviços de saúde para projetar ações apropriadas dirigidas a grupos específicos da população com o objetivo de melhorar o estado nutricional da população infantil.

Descritores: Estado nutricional. Fatores sociodemográficos. Saúde infantil.

GONZALEZ P., G.J. \& VEGA L., M.G. [Sociodemographic conditions and nutritional status of children under one year old in areas adjacent to Guadalajara, México]. Rev. Saúde Pública, 28: 268-76, 1994. A random population sample was studied with a view to determining the proportion of children of less than on year of age whose weigth is less than the minimum adequate weigth (MAW) for their age, as well as to identifying factors of a socio-demographic nature that could be associated with this problem and the socio-sanitary importance of these factors. The sample studied was of children of less than one year of age, born between 05/01/1990 and 04/ $30 / 1991$, whose mothers were entited to the services of the Mexican Institute of Social Security, in Tlaquepaque and Tonalá, municipalities adjacent to the Metropolitan Zone of Guadalajara, the second most important city in Mexico. The ponderal assessment of the infants was performed on the basis of tables which the LatinAmerican Perinatology Center had developed for this purpose. Through the use of logistic regression, Odds Ratios (OR) were estimated, with $90 \%$ confidence intervals $(\mathrm{CI})$. The model thus prepared was adjusted by means of the $\mathrm{H}^{*}$ statistics. Likewise, the population's attributable risk (PAR) was calculated, as well as the probability that a child would not reach the MAW for its age, in the presence or abscence of particular risk factors. The results indicate that nearly one fifth of the infants studied do not reach MAW. The work shows that a statistical association exists between the mother's being less than 20 years old $(\mathrm{OR}=2.53, \mathrm{CI} 1.03-6.22)$ and her belonging to the "working class" (OR=2.28, $\mathrm{CI} 1.0 \mathrm{l}$ 5.21), and the infant's not reaching MAW, and the social effects of these factors (PAR $=26.4 \%$ and $36.7 \%$, respectively). In the presence of all the factors analyzed, the probability of a child's not reaching MAW is of 0.85 and, in particular, in the presence of the given risk factors, the probability is of 0.41 . These data suggest guidelines for health services to design appropriate action targeted at specific population groups with a view to improving the nutritional state of the infant population.

Keywords: Nutritional status. Sociodemographic factors. Child health.

\section{Referencias Bibliográficas}

1. ATALAH, E. et al. Necesidades y expectativas familiares de servicios preescolares en el urbano pobre de Santiago. In: Taller Latinoamericano sobre Nutrición y Salud en Áreas Urbanas, 20, México, DF, 1992. Libro de resumenes. México, DF, 1992.

2. ATEHORTÚA, P.W. et al. Estudio de base "Nutrición y Salud" en zonas urbanas marginales de Cúcuta, Colombia In: Taller Latinoamericano de Nutrición y Salud en Áreas Urbanas, $2^{\circ}$, México, DF, 1992. Libro de resumenes. México, DF, 1992.

3. AVILA CURIEL, A. Humbre, desnutrición y sociedad. México, DF, Edit. Univ. de Guadalajara, 1990.

4. BARROS, F.C. et al. Bajo peso al nacer en el municipio de Pelotas, Brasil: factores de riesgo, Bol. Oficina Sanit. Panam., 102:541-52, 1987.

5. CASANUEVA, E. et al. Adolescencia y embarazo: impacto de una intervención psicosocial sobre la nutrición materna. In: Taller Latinoamericano sobre Nutrición y Salud en Áreas Urbanas, $2^{\circ}$, México, DF, 1992. Libro de resumenes. México, DF, 1992.

6. CRAVIOTO, J. et al. La desnutrición protéico calórica y el desarrollo psicobiológico del niño. Bol. Oficina Sanit. Panam., 45: 1966.

7. GÓMEZ, F. Desnutrición. Bol.Med.Hosp.Inf.Méx., 3(4): 1946.

8. GONZÁLEZ PEREZ, G. et al. Factores socioeconómicos asociados a la mortalidad postneonatal en Cuba. Rev.Saúde Pública, 24: 134-43, 1990.

9. INSTITUTO NACIONAL DE ESTADÍSTICAS, GEOGRAFÍA E INFORMÁTICA. Jalisco; resultados definitivos. México, DF, 1992. $11^{\circ}$ Censo de Población y Viviendas, 1990

10. JORDÁN, J.R. El lactante de 0-2 años: antropometría y crescimiento. In: Cusminsky, M.; Moreno, E.M.; Suárez Ojeda, E.N., ed. Crescimiento y desarrollo: he. 
chos y tendencias. Washington, D.C, Organización Panamericana de la Salud, 1988. p. 184-209. (OPS - Publicación Científica, 510).

11. LEÓN, D. Logit regression analysis for personal computers. Bangkok, The Population Council, Asia Regional Office, 1985.

12. LEMESHOW, S. \& HOSMER, D.W. A review of goodness of fit statistics for use in the development of logistic regression model. Am.J.Epidemiol., 115:92-106, 1982.

13. LEVIN, J. Fundamentos de estadística para la investigación social. México, DF, Ed. Harla, 1979.

14. LILIENFIELD, et al. Fundamentos de epidmiologia. MéxiCo, DF, Addson-Wisley, 1987.

15. MARTELL, M. \& BELITZKY, R. Tecnología apropiada: tablas y curvas para la evaluación del crecimiento en los dos primeros años de vida. Salud Perinatal, 1: 1984.

16. MUÑOZ, L.M. Crecimiento infantil en zonas marginales urbanas de Costa Rica. In: Taller Latinoamericano sobre Nutrición y Salud en Áreas Urbanas, $2^{\circ}$, México, DF, 1992. Libro de resumenes. México, DF, 1992.

17. MURILLO, S. Nutrición urbana en preescolares de Costa Rica. In: Taller Latinoamericano de Nutrición y Salud Ca Áreas Urbanas, $2^{\circ}$, México, DF, 1992. Libro de resumenes. México, DF, 1992.
18. PIMENTEL, V.A.F. et al. El crecimiento de los niños alimentados exclusivamente con leche materna durante los seis primeros meses de vida. Bol. Oficina Sanit. Pan. am., 110: 311-8, 1991.

19. PUFFER, R.R. \& SERRANO, C.V. Caracteristicas del peso al nacer. Washington, D.C., Organización Panamericana de la Salud, 1988. (()PS - Publicación Científica, 504).

20. RAMOS GALVÁN. R. Consecuencias de la desnutrición crónica en los grupos humanos. Gac.Med.Méx., 111:297, 1976.

21. ROHTMAN, K. J. Modern epidemiology. Boston, Little, Brown and Company, 1986.

22. SCATENA VILLA, T.C. \& ROTTER PELÁ, N.T. Aleitamento materno e suplementaçảo alimentar. Bol. Oficina Sanit Panam., 106:108-16, 1989.

23. SEPÚL VEDA AMOR, J, et al. Estado nutricional de preescolares y mujeres en México: resultados de una encuesta probabilística nacional. Gac.Med.Méx., 126:207-24, 1990.

24. SILVA, L.C. Métodos estadísticos para la investigación epidemiológica. Bilbao, Instituto Vasco de Estadística, 1988.

Recebido para publicação ent /5.1.1993

Reapresentado em 9.12.1993

aprovado para publicação em 8.6.1994 